\title{
ARTICLE
}

\section{A new recombinant MS-superoxide dismutase alleviates 5 -fluorouracil-induced intestinal mucositis in mice}

\author{
Xiao-xia Yan ${ }^{1,2}$, Hai-long $\mathrm{Li}^{3,4}$, Yi-ting Zhang ${ }^{1,2}$, Shou-yan $\mathrm{Wu}^{1,2}$, Heng-lei $\mathrm{Lu}^{1}$, Xiao-lu Yu ${ }^{1,2}$, Fan-guo Meng ${ }^{3,4}$, Jian-hua Sun ${ }^{1}$ and \\ Li-kun Gong ${ }^{1,2}$
}

Intestinal mucositis is a common side effect of anticancer regimens that exerts a negative impact on chemotherapy. Superoxide dismutase (SOD) is a potential therapy for mucositis but efficient product is not available because the enzyme is degraded following oral administration or induces an immune reaction after intravascular infusion. Multi-modified Stable Anti-Oxidant Enzymes ${ }^{\oplus}$ (MS-AOE ${ }^{\oplus}$ ) is a new recombinant SOD with better resistance to pepsin and trypsin. We referred it as MS-SOD to distinguish from other SODs. In this study we investigated its potential to alleviate 5-FU-induced intestinal injury and the mechanisms. An intestinal mucositis model was established in C57/BL6 mice by 5-day administration of 5-FU (50 mg/kg every day, ip). MS-SOD (800 IU/10 g, ig) was given once daily for 9 days. 5-FU caused severe mucositis with intestinal morphological damage, bodyweight loss and diarrhea; MS-SOD significantly decreased the severity. 5-FU markedly increased reactive oxygen species (ROS) and inflammatory cytokines in the intestine which were ameliorated by MS-SOD. Furthermore, MS-SOD modified intestinal microbes, particularly reduced Verrucomicrobia, compared with the 5-FU group. In Caco2 cells, MS-SOD (250-1000 U/mL) dosedependently decreased tBHP-induced ROS generation. In RAW264.7 cells, MS-SOD (500 U/mL) had no effect on LPS-induced inflammatory cytokines, but inhibited iNOS expression. These results demonstrate that MS-SOD can scavenge ROS at the initial stage of injury, thus play an indirect role in anti-inflammatory and barrier protein protection. In conclusion, MS-SOD attenuates 5FU-induced intestinal mucositis by suppressing oxidative stress and inflammation, and influencing microbes. MS-SOD may exert beneficial effect in prevention of intestinal mucositis during chemotherapy in clinic.

Keywords: manganese superoxide dismutase; 5-fluorouracil; chemotherapy; intestinal mucositis; diarrhea; oxidative stress; cytokines; intestinal microbes

Acta Pharmacologica Sinica (2020) 41:348-357; https://doi.org/10.1038/s41401-019-0295-8

\section{INTRODUCTION}

Malignant tumors are a serious threat to life, and chemotherapy remains one of the main therapies used to treat malignant tumors. However, chemotherapy drugs not only eliminate tumor cells but also kill a large number of normal tissue cells, especially rapidly differentiating intestinal mucosal cells, which leads to intestinal mucosal injury, known as mucositis [1, 2]. The incidence of intestinal mucosal injury caused by chemotherapy is $\sim 40 \%$, and among these cases, the incidence of intestinal mucosal injury from 5-fluorouracil (5-FU) and methotrexate is as high as 90\% [1]. The symptoms of mucositis include diarrhea and inflammation. Diarrhea can cause dehydration and electrolyte imbalance and even be life-threatening. Bacterial infection caused by mucosal inflammation leads to severe systemic infections in patients $[3,4]$. However, current treatments for chemotherapy-induced mucositis, such as amifostine, loperamide, octreotide, sucralfate and anti-inflammatory agents, have limited effects [5-9]. Thus, an urgent need to develop novel therapeutic strategies that target intestinal mucositis exists.

Although the pathogenesis of intestinal mucositis is not completely understood, it has been reported that reduced cellular proliferation and increased apoptosis in the intestinal epithelium cause the loss of intestinal structure and function, primarily via villus shortening and crypt ablation [10]. Furthermore, chemotherapy initiates the release of reactive oxygen species (ROS) and proinflammatory cytokines, which play an indirect role in amplifying the intestinal damage $[10,11]$.

The administration of exogenous superoxide dismutase (SOD) can eliminate ROS produced in the initial stage of mucosal injury [12], thereby improving or delaying injury; therefore, SOD is a potential therapeutic drug. Since the 1990s, products containing SOD have gradually been introduced onto the market. For example, Palosein ${ }^{\oplus}$ (Oxis International Inc., USA), which is extracted from bovine blood, was once marketed in Europe for the treatment of inflammation and prevention of radiation-induced side effects, but this drug was withdrawn due to immune reactions [13]. GliSODin (Isocell Pharma, France), which is a combination of cantaloupe extract and gliadin, was targeted to the intestine, but fragments generated by its hydrolysis caused allergic reactions [14]. Although many researchers have modified SOD via genetic engineering to overcome these defects, many problems still exist, e.g., ordinary SOD products have a

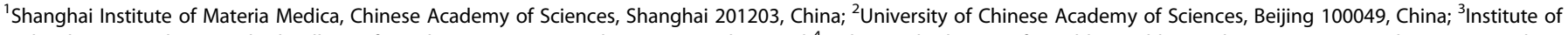

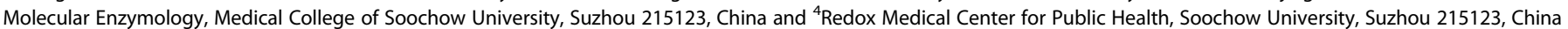
Correspondence: Jian-hua Sun (jhsun@cdser.simm.ac.cn) or Li-kun Gong (lkgong@cdser.simm.ac.cn)

These authors contributed equally: Xiao-xia Yan, Hai-long Li

Received: 31 January 2019 Accepted: 6 August 2019

Published online: 10 September 2019 
poor resistance to high temperature or long-term storage in the drug preparation process. Even worse, because SOD is a macromolecule, transdermal absorption is limited, and SOD is easily digested and degraded by proteases in the gastrointestinal tract when orally administered.

MS-Superoxide Dismutase (MS-SOD) [15] is a new and highly stable manganese SOD obtained from a mutant hightemperature-resistant SOD strain. Compared with wild-type SOD and ordinary heat-resistant SODs, MS-SOD is more stable and shows better resistance to pepsin and trypsin, making MS-SOD a potential candidate for clinical development. MS-SOD is thus expected to be a new treatment for intestinal mucositis.

In addition, MS-SOD has been prescribed to treat gastrointestinal disorders and shown promise in alleviating the symptoms of 2,4,6-trinitrobenzene sulfonic acid (TNBS)-induced colitis in zebrafish by markedly modulating inflammation and ulceration [15]. However, no further research on MS-SOD has been conducted in rodents, and whether MS-SOD is effective against chemotherapy-induced intestinal mucositis remains unknown. Therefore, in the current study, the effect of MSSOD on 5-FU-induced intestinal mucositis was investigated in mice. MS-SOD treatment was found to reduce intestinal injury by attenuating morphological damage, bodyweight loss, and diarrhea. The influence of MS-SOD on oxidative stress, inflammation and intestinal microbes was further explored.

\section{MATERIALS AND METHODS}

\section{Animals and experimental design}

This study was conducted in strict accordance with the recommendations in the Guide for the Care and Use of Laboratory Animals by the National Institutes of Health. The protocols were approved by the Committee on Ethics of Shanghai Institute of Materia Medica. Briefly, 6- to 8-week-old male specific pathogenfree C57/BL6 mice weighing $20-24 \mathrm{~g}$ were obtained from Shanghai Laboratory Animal Co. (Shanghai, China). All mice were maintained in plastic cages with free access to food and water and housed at $20-25^{\circ} \mathrm{C}$ with a $12 \mathrm{~h}$ light/dark cycle. The mice were fed for one week to acclimate them to the environment before the experiment. The animals were distributed as follows: Group IControl (untreated), Group II-5-FU (vehicle alone), and Group III -5 -FU + MS-SOD. Intestinal mucositis was induced in Groups II and III via the intraperitoneal administration of $50 \mathrm{mg} / \mathrm{kg} 5-\mathrm{FU}$ (Sigma-Aldrich, St Louis, MO, USA) for 5 days [16]. Mice in Groups II and III were also treated orally (by gavage) with water or MSSOD, respectively, for 9 days, while vehicle was administered to the mice in Group I. On the 4th, 6th, 8th and 10th days, the animals were sacrificed ( $n=6 /$ group). Body weights, intestine lengths, and the results of diarrhea assessment and histopathological analysis were recorded for all animals. In the other assays, the animals were sacrificed on day 6 .

\section{Chemicals}

5 -FU was dissolved in a $5 \%$ glucose solution at a concentration of $5 \mathrm{mg} / \mathrm{mL}$. MS-SOD (Redox, Suzhou, China, $4200 \mathrm{U} / \mathrm{mg}$ ) was dissolved in water at a concentration of $1.9 \mathrm{mg} / \mathrm{mL}$. All drugs were prepared immediately before use and administered via intraperitoneal (i.p.) injection or intragastrically (i.g.) at a volume of $0.1 \mathrm{~mL} / 10 \mathrm{~g}$ of body weight.

\section{Assessment of body weight and diarrhea}

The animals were weighed daily during the induction process, and the results are expressed as variation in body weight (\%) relative to the initial weight at the beginning of the experiment. Diarrhea severity was scored using the following scale: 0 , normal (normal stool); 1, minimal (soft stool); 2, slight (slightly wet and soft stool); 3, moderate (wet and unformed stool with moderate perianal staining of the coat); or 4, severe (watery stool with severe perianal staining of the coat). The number of diarrhea scores of 0 to 4 and the average diarrhea score were used to evaluate the severity of diarrhea [17]. The first day on which the severity of diarrhea was observed was treated as the "onset of diarrhea." The bleeding score was detected using fecal occult blood (OB) test paper (BASO Diagnostics Inc., Zhuhai, China) [18].

Intestinal length measurement and histopathological analysis The intestine of each animal was dissected, and its length was measured. Then, the small intestine and large intestine were washed with phosphate-buffered saline (PBS) and fixed in $10 \%$ phosphate-buffered formalin $(\mathrm{pH}$ 7.4) for histopathological evaluation. Tissue samples were then dehydrated and embedded in paraffin, sectioned ( $4 \mu \mathrm{m}$ thickness), and stained with hematoxylin and eosin (H\&E). Histological lesions were scored by a pathologist at the Center for Drug Safety Evaluation and Research at the Shanghai Institute of Materia Medica. Each slide was subsequently photographed using a light microscope (Axio Scope A1, Carl Zeiss, Jena, TH, Germany) with a $\times 20$ objective lens.

Determination of mRNA expression via quantitative PCR

The small intestine tissue was washed with PBS, and total RNA was extracted from 20-mg samples using TRIzol (Invitrogen, Carlsbad, CA, USA) according to the manufacturer's instructions. A PrimeScript RT kit (TaKaRa, Osaka, Japan) was used for cDNA synthesis using quantitative real-time polymerase chain reaction (qPCR) with a Rotor-Gene Qplex-HRM system (Qiagen, Redwood City, CA, USA) using SYBR ${ }^{{ }^{\circ}}$ Premix Ex Taq ${ }^{\mathrm{TM}} \|$ (TaKaRa). The specific primer sequences used to amplify GAPDH, IL-6, IL-17a, IL-22, CXCL1, CXCL2, Claudin-2 and Occludin are listed in Table 1. All mRNA expression levels were calculated using the comparative $C T(\Delta \Delta C T)$ method (normalized to the mean value of the control group).

Preparation of intestinal tissue homogenates

A small intestinal segment was removed from each animal and stored at $-20^{\circ} \mathrm{C}$ for subsequent analysis. After thawing, $100 \mathrm{mg}$ of each intestinal sample was washed in PBS (pH 6.0) and transferred to a tube, and $1 \mathrm{~mL}$ of PBS was added to each tube. The tissues were then homogenized using a Scientz-48 tissue grinder (Ningbo Scientz Biotechnology Co., Ningbo, China) and then centrifuged at $400 \times g$ at

Table 1. Primer sequences for quantitative real-time $P C R$ analysis

\begin{tabular}{lll}
\hline Gene & Primer sequences & \\
\hline GAPDH & Forward $\left(5^{\prime}-3^{\prime}\right)$ & AACTTTGGCATTGTGGAAGG \\
& Reverse $\left(3^{\prime}-5^{\prime}\right)$ & ACACATTGGGGGTAGGAACA \\
Nrf2 & Forward $\left(5^{\prime}-3^{\prime}\right)$ & CTTTAGTCAGCGACAGAAGGAC \\
& Reverse $\left(3^{\prime}-5^{\prime}\right)$ & AGGCATCTTGTTTGGAATGTG \\
Occludin & Forward $\left(5^{\prime}-3^{\prime}\right)$ & ATGGCAAGCGATCATACCC \\
& Reverse $\left(3^{\prime}-5^{\prime}\right)$ & TTCCTGCTTTCCCCTTCG \\
IL-6 & Forward $\left(5^{\prime}-3^{\prime}\right)$ & GACTTCCATCCAGTTGCCTTCT \\
& Reverse $\left(3^{\prime}-5^{\prime}\right)$ & AGACAGGTCTGTTGGGAGTGTA \\
IL-17 $\alpha$ & Forward $\left(5^{\prime}-3^{\prime}\right)$ & GTGAAGGCAGCAGCGATCAT \\
& Reverse $\left(3^{\prime}-5^{\prime}\right)$ & GCGCCAAGGGAGTTAAAGACT \\
IL-22 & Forward $\left(5^{\prime}-3^{\prime}\right)$ & AGCTGCCTGCTTCTCATTGC \\
& Reverse $\left(3^{\prime}-5^{\prime}\right)$ & GACGATGTATGGCTGCTGGAA \\
IL-1 $\beta$ & Forward $\left(5^{\prime}-3^{\prime}\right)$ & GAAATGCCACCTTTTGACAGTG \\
& Reverse $\left(3^{\prime}-5^{\prime}\right)$ & TGGATGCTCTCATCAGGACAG \\
CXCL1 & Forward $\left(5^{\prime}-3^{\prime}\right)$ & GCTCCCTTGGTTCAGAAAATTG \\
& Reverse $\left(3^{\prime}-5^{\prime}\right)$ & TCACCAGACAGGTGCCATCA \\
CXCL2 & Forward $\left(5^{\prime}-3^{\prime}\right)$ & CCTGCCAAGGGTTGACTTC \\
& Reverse $\left(3^{\prime}-5^{\prime}\right)$ & TTTTGACCGCCCTTGAGAGT
\end{tabular}


$4{ }^{\circ} \mathrm{C}$ for $15 \mathrm{~min}$. The obtained supernatants were used in assays. The experiments were carried out in triplicate.

ROS and detection of total antioxidant capacity Intestinal tissue homogenates were centrifuged, washed in PBS and loaded with $50 \mu \mathrm{M} 2^{\prime}, 7^{\prime}$-dichlorofluorescein-diacetate (DCFHDA) for $10 \mathrm{~min}$ at $37^{\circ} \mathrm{C}$ to determine the ROS content. Fluorescence was detected with a BIOTEK S4 ML FPTAD instrument (BioTek, Milton, VT, USA). The total antioxidant capacity was detected using a T-AOC assay kit (Beyotime, Nanjing, China). Moreover, a mouse myeloperoxidase (MPO) ELISA kit (Cusabio, Wuhan, China) was used to detect MPO content.

\section{Western blotting}

Mouse intestinal tissues $(30 \mathrm{mg}$ ) were lysed with radioimmunoprecipitation assay buffer (Beyotime). Protein lysates were separated on $10 \%$ SDS-PAGE gels and electrophoretically transferred to polyvinylidene fluoride (PVDF) membranes (Millipore, Billerica, MA, USA). The membranes were blocked with $5 \%$ skim milk and incubated overnight at $4{ }^{\circ} \mathrm{C}$ with antibodies against occludin (Abcam, Cambridge, MA, USA), nuclear factor (erythroid-derived 2)-like 2 (Nrf-2; Enzo Life Sciences, Inc., Farmingdale, NJ, USA) and heme oxygenase 1 (HO-1; Enzo Life Sciences, Inc.). The membranes were then incubated with horseradish peroxidase-conjugated secondary antibodies (Jackson ImmunoResearch Laboratories, Inc., West Grove, PA, USA). Protein band chemiluminescence was detected using an ECL Plus immunoblot detection system (Millipore) and analyzed by densitometry. The housekeeping gene was detected on the same membrane using a monoclonal rabbit anti-GAPDH antibody (1:5000 dilution; ab9485, Abcam) to confirm equivalent protein loading.

Bacterial DNA extraction/sequencing and analysis of bacterial $16 \mathrm{~S}$ rDNA

Fecal samples were suspended in PBS and centrifuged at $14000 \times$ $g$, and the pellets were resuspended in $100 \mathrm{mM}$ Tris- $\mathrm{HCl}(\mathrm{pH} 9.0)$ containing $40 \mathrm{mM}$ EDTA and $1 \%$ sodium dodecyl sulfate. The suspension was mixed with buffer-saturated phenol and 0.1-mm glass beads and shaken at $800 \times g$ for $10 \mathrm{~s}$. After centrifugation at $14000 \times g$ for $5 \mathrm{~min}$, the supernatant was collected, extracted with phenol-chloroform and precipitated with isopropanol. The resulting DNA pellet was washed with $70 \%$ ethanol, dried and dissolved in $10 \mathrm{mM}$ Tris- $\mathrm{HCl}(\mathrm{pH} 8.0)$ with $1 \mathrm{mM}$ EDTA. The composition of the intestinal microbial community was analyzed based on the V3V4 region in 16S rRNA (Majorbio, Shanghai, China).

In vitro cell system tests

Human colorectal adenocarcinoma (Caco2) cells were treated with tert-butyl hydroperoxide (tBHP) to induce the generation of ROS. In brief, 31.25 $\mu \mathrm{M}$ tBHP was added to wells containing Caco2 cells for $1 \mathrm{~h}$, and MS-SOD at the indicated concentrations was also added simultaneously (Supplementary Fig. S1). Cells were incubated for 30 min with $25 \mu \mathrm{M}$ DCFH-DA, and the ROS content was then detected with the fluorescence method described above.

RAW264.7 cells were preincubated with $500 \mathrm{U} / \mathrm{mL}$ MS-SOD for $6 \mathrm{~h}$ and then stimulated with $10 \mathrm{~g} / \mathrm{mL}$ LPS to induce the generation of inflammatory cytokines. Samples were collected $6 \mathrm{~h}$ after LPS stimulation, and IL-1 $\beta$, IL-6, TNF- $\alpha$ and inducible nitric oxide synthase (iNOS) mRNA expression levels were measured as described above.

\section{Statistical analysis}

Data were analyzed and graphed using GraphPad Prism software (version 5.03; GraphPad Software, Inc., La Jolla, CA, USA) and are expressed as the means \pm SEM. Student's $t$-tests were used to determine the significance of differences. A Wilcoxon rank-sum test was used to analyze data at the phylum level. $P<0.05$ indicated statistical significance.

\section{RESULTS}

MS-SOD reduced body weight loss and diarrhea induced by 5-FU Repeated 5-FU administration $(50 \mathrm{mg} / \mathrm{kg}$ ) for 5 days (days $1-5$ ) decreased the body weights of the animals (Fig. 1a, b). Significant body weight loss was observed on day 3 and reached $16 \%$ reduction on day 5 . The body weights gradually recovered after 5 FU withdrawal. Once-daily administration of MS-SOD (800 IU/10 g) until day 9 reduced body weight loss during or after 5-FU treatment, and this effect was significant on days 5, 8 and 9. Diarrhea and fecal blood are common gastrointestinal symptoms of mucositis following chemotherapy [19]. No abnormalities were observed in the control group. However, 5-FU treatment caused an elevation in the diarrhea score on day 4, and these symptoms persisted after 5-FU withdrawal. MS-SOD treatment had an antidiarrheal effect (Fig. 1c). Consistent with 5-FU-induced diarrhea, fecal blood was also observed on day 4 , and the fecal blood score was worsened daily until sacrifice. A reduction in the fecal blood score was also found in the MS-SOD treatment group (Fig. 1d).

\section{MS-SOD attenuated intestinal injury}

The intestinal damage induced by chemotherapy is partially manifested by a reduction in intestinal length [20]. In the current study, the lengths of both the small intestine and colon were reduced in 5-FU-treated animals, and a significant reduction in small intestine and colon length was observed on day 6. Treatment with MS-SOD prevented this decrease (Fig. 2b). Furthermore, to evaluate 5-FUinduced mucosal damage at the microscopic level, H\&E-stained sections of the small intestine and colon were examined. Villus height and crypt depth were reduced throughout the small intestine in all 5FU-treated groups compared with those in the control group. Mucosal erosion and subacute inflammation occurred in the colons of mice in the 5-FU-treated groups. However, in the MS-SOD-treated mice, the villus height, crypt depth, mucosal erosion and subacute inflammation in the intestine were improved compared with those in mice in the 5-FU group, and this difference was significant on day 6 . Furthermore, the mucosa was thicker following MS-SOD treatment compared with that in the 5-FU group (Fig. 2a). The reduction in intestine length observed with 5-FU treatment was gradually reversed in the mice after 5-FU withdrawal.

\section{MS-SOD increased occludin expression}

Occludin is an important protein for tight junction stability and barrier function [21]. Previous studies have indicated that mice that do not express occludin show morphological instability in several epithelial tissues and chronic inflammation [21]. The occludin mRNA (Table 1) and protein levels in the small intestine were decreased in mice after 5 days of treatment with 5-FU, which was in accordance with the observed microscopic damage. However, the occludin mRNA and protein expression levels in MS-SOD-treated mice were increased compared with those in the 5-FU group (Fig. 3a).

\section{MS-SOD decreased cytokine expression}

After treatment with 5-FU, the expression of IL-6, IL-17a, IL-22, CXCL1 and CXCL2 mRNA in the small intestine was upregulated $[22,23]$. The concentration of IL- 6 in the serum was also found to be increased. IL- 6 is one of the main proinflammatory cytokines. $\mathrm{IL}-17 \mathrm{a}$ and IL-22, which are signature cytokines of $\mathrm{T}$ helper cell 17 , might initiate and amplify multiple proinflammatory pathways and have also been reported to be related to Crohn's disease and ulcerative colitis. It has been reported that 5-FU could increase CXCL1 and CXCL2 expression in the intestinal tracts of mice, which may be related to the degree of inflammation and diarrhea [24]. These cytokines were downregulated in MS-SOD-treated mice (Fig. 3c, d). Moreover, a significant increase in MPO content was found in the 5-FU group $(P<0.05$, Fig. $3 b)$, but MS-SOD did not significantly decrease the MPO level after intervention. 
a
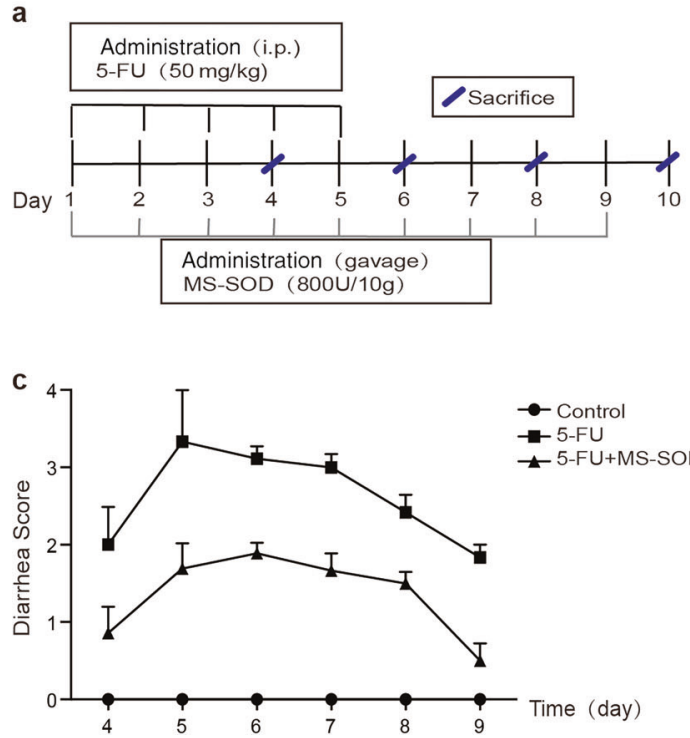
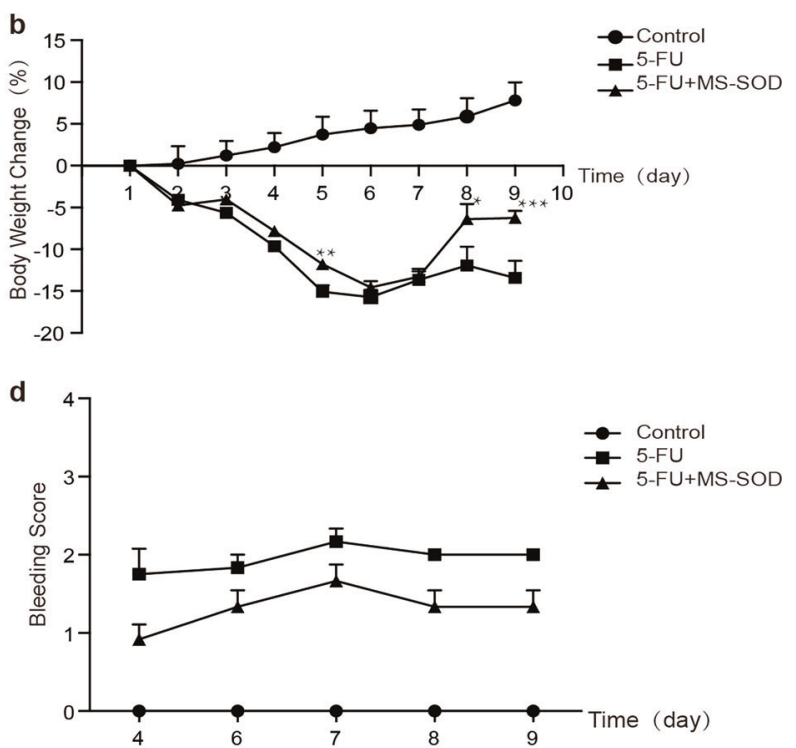

Fig. 1 Schedule for the administration and effects of MS-SOD on 5-FU-induced changes in body weight, diarrhea and fecal blood score in mice. MS-SOD (800 IU/10 g, i.g.) or vehicle (diluted water, i.g.) was administered from day 1 to day 9, and 5-FU (50 mg/kg, i.p.) or vehicle (saline, i.p.) was administered from day 1 to day 5. Animals were sacrificed on days $4,6,8$ and 10 ( $n=6 /$ group) (a). Overview of the study setup. The body weight of mice treated with vehicle or MS-SOD was recorded daily (b), and diarrhea and fecal blood scores (c, $\mathbf{d})$ were recorded from day 4. Significant body weight loss induced by 5-FU was observed on day 3, and MS-SOD significantly reduced this loss on days 5 , 8 and 9 . Diarrhea and the fecal blood score were worsened daily in the vehicle group, but MS-SOD treatment reduced the score each day. The data are presented as the means \pm SEM $(n=6) ;{ }^{*} P<0.05,{ }^{* *} P<0.01$, and ${ }^{* * *} P<0.001$ according to unpaired $t$-tests

a

lleum

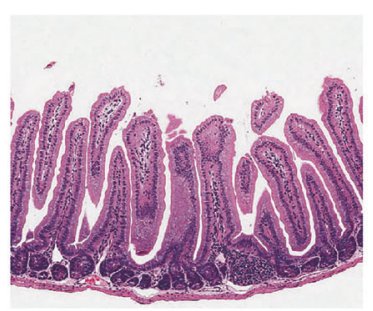

b
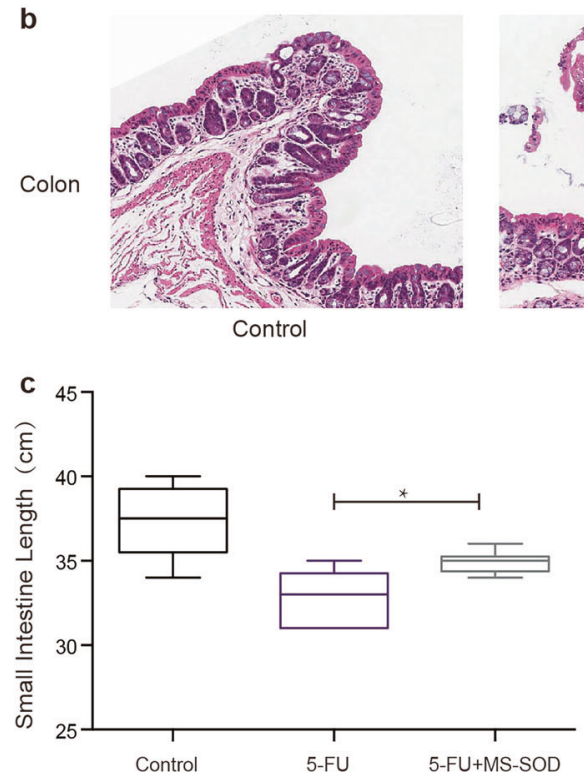
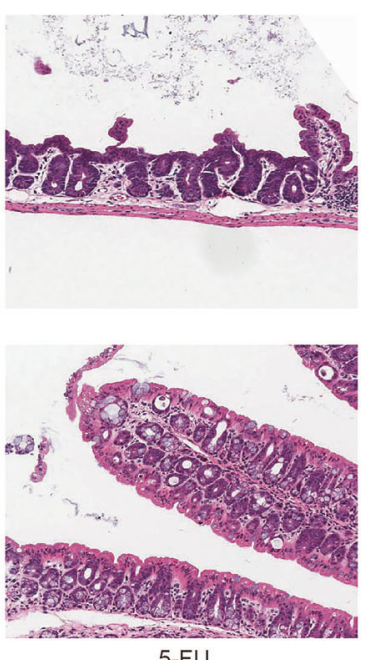

$5-F U$

d

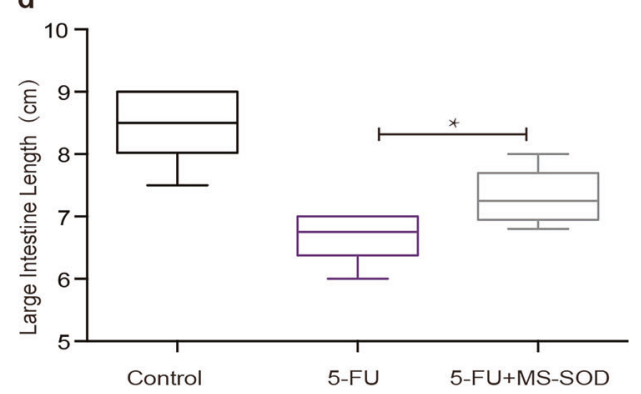

Fig. 2 Histological changes and shortening of the small intestine and colon after 5-FU administration and MS-SOD treatment. Five days after 5-FU challenge, the small intestine (a) and colon (b) from control, 5-FU, and 5-FU + MS-SOD mice were sectioned and prepared for H\&E staining $(\times 200)$, and shortening of the small intestine (c) and colon (d) was assessed to evaluate the severity of mucositis. Villus height and crypt depth reduction in the small intestine occurred in the 5-FU group, accompanied by mucosal erosion and subacute inflammation in the colon. Additionally, the intestinal length was decreased due to atrophy induced by 5-FU, but MS-SOD treatment improved the symptoms. The data are presented as the means \pm SEM $(n=6) ;{ }^{*} P<0.05$ according to unpaired $t$-tests 
a

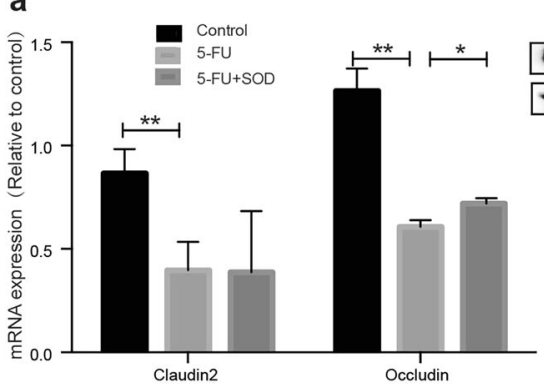

C IL-6
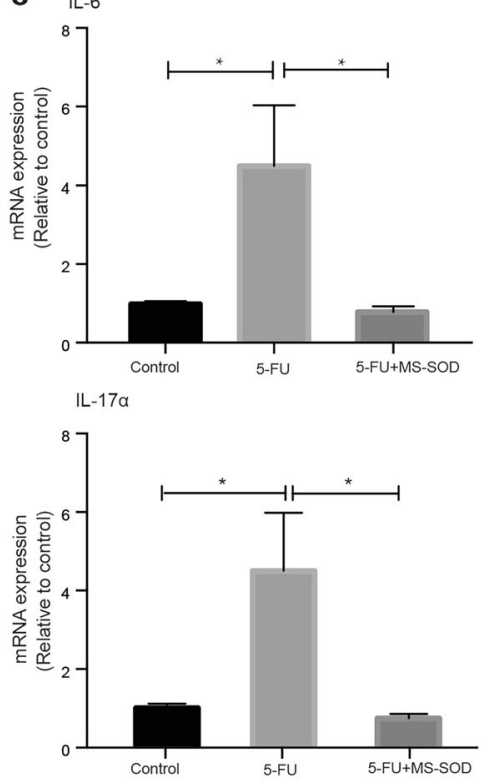
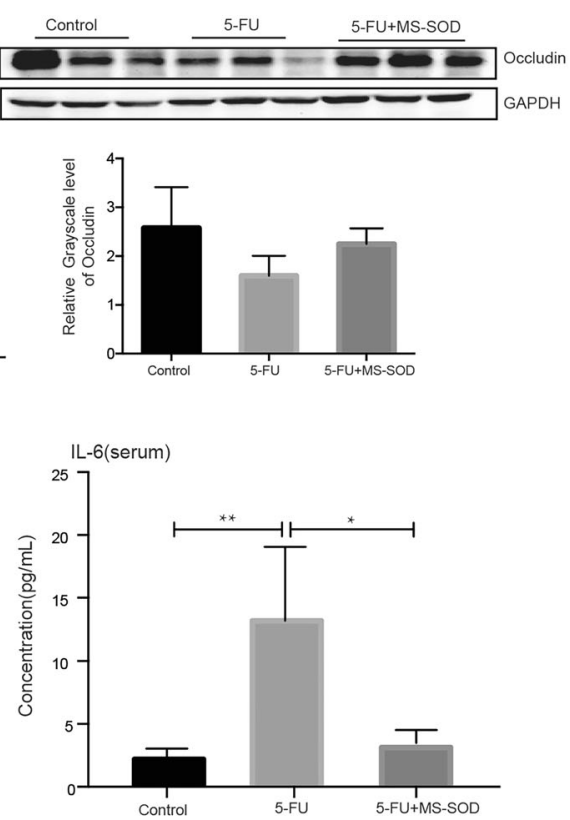

IL-22

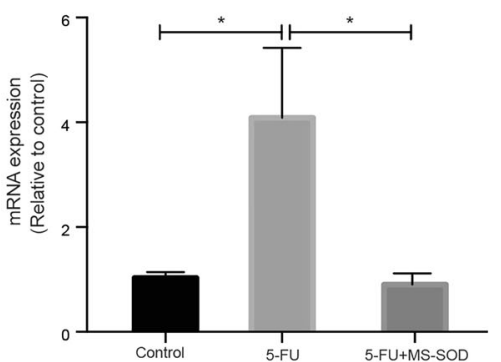

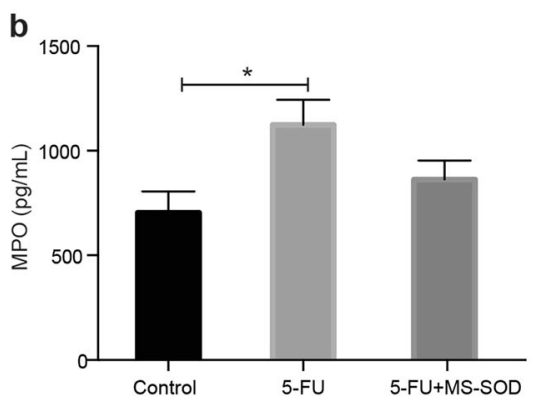

d
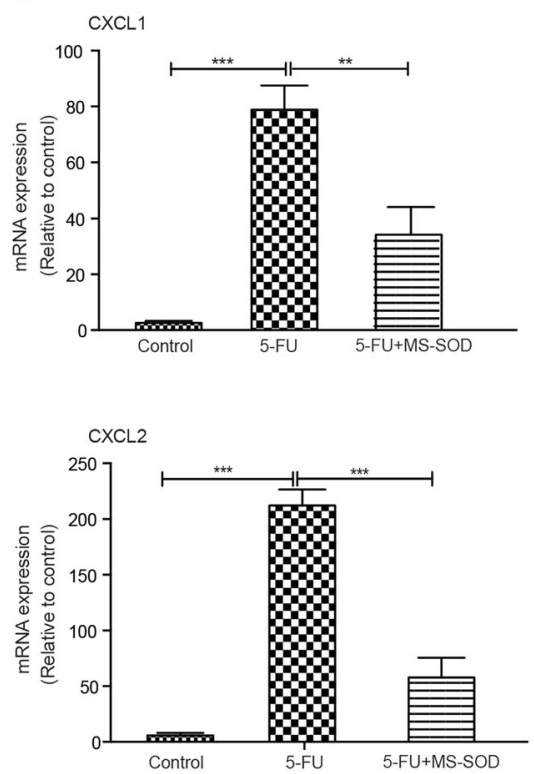

Fig. 3 Effects of MS-SOD on tight junctions and cytokines in the small intestine after 5-FU treatment. qPCR analysis was performed to measure the transcription levels of occludin (a), IL-6, IL-17 $\alpha$, IL-22 (c), CXCL1 and CXCL2 (d). Western blotting was used to measure the translation level of occludin (a). An ELISA was used to measure the IL- 6 concentration in serum (c). Both occludin mRNA and protein levels in the small intestine were decreased in mice after 5 days of 5-FU injection but were increased in the MS-SOD group. The mRNA expression levels of the main pro-inflammatory cytokines, IL-6, IL-17 $\alpha$ and IL-22 as well as the chemokines CXCL1 ans CXCL2, were upregulated in the 5FU group, but these cytokines were downregulated in MS-SOD-treated mice. The serum IL- 6 concentration was in accordance with the mRNA level. ELISA method was used for MPO content measure. MPO content in intestinal tissue homogenates was significantly increased in the 5-FU group, but did not decrease in MS-SOD group (b). The data are shown as the means \pm SEM $(n=6)$. ${ }^{*} P<0.05,{ }^{* *} P<0.01,{ }^{* * *} P<0.001$. In a, the repeated blots in each group indicate three individuals

MS-SOD reduced ROS content and elevated antioxidant capacity Following 5-FU injection, ROS were released in the small intestine, and their levels were significantly increased in the 5FU group compared with those in the control [11]. However, a reduction in ROS was observed in MS-SOD-treated mice (Fig. 4a). The total antioxidant capacity in the small intestine was decreased in the 5-FU group. MS-SOD treatment elevated the antioxidant capacity on day 3 , but the effect was not significant later in the experiment (Fig. 4b). In addition, when we examined the groups after 5 days of treatment, we found that 5 -FU treatment decreased Nrf2 mRNA and protein expression in the small intestine, but MS-SOD increased both Nrf2 and heme oxygenase-1 (HO-1) expression in the small intestine (Fig. 4c) [25]. Nrf2, a transcription factor and the master regulator of numerous antioxidants and antiapoptotic proteins (including HO-1), accumulates in the nucleus during oxidative injury. In addition, tissue SOD is a downstream antioxidant factor of activated Nrf2, and SOD activity was upregulated in MS-SODtreated mice (Fig. 4d). Taken together, these results suggest that MS-SOD partially exerts its beneficial effects via a reduction in ROS accumulation and oxidative stress accompanied by activation of the antioxidant pathway.
MS-SOD had an effect on intestinal microbes

The gastrointestinal tract harbors a wide variety of microorganisms known as intestinal microbes. Studies have found that 5-FU administration changes the intestinal microbes in mice and that these changes are one of the factors associated with intestinal mucosal injury [26]. In this study, second-generation sequencing was used to determine and quantify the intestinal flora in mice. At the phylum level, the abundance of Bacteroides decreased, but the abundance of Lachnospiraceae and Verrucomicrobia increased in the 5-FU group compared with that in the control group. MS-SOD reversed these effects (Fig. $5 \mathrm{a}$ ). The specific data are shown in Table 2. We also performed the analysis at the genus level among the three groups (Fig. 5b).

MS-SOD inhibited tBHP-induced ROS generation in Caco-2 cells As shown by the results in Fig. 6, MS-SOD decreased ROS generation in $\mathrm{CacO} 2$ cells in a dose-dependent manner, which indicated that MSSOD can dose-dependently inhibit tBHP-induced ROS generation.

MS-SOD inhibited LPS-induced ROS generation in RAW264.7 cells MS-SOD $(500 \mathrm{U} / \mathrm{mL})$ had no significant effect on inflammatory cytokines but inhibited the expression of iNOS mRNA (Fig. 7). iNOS 
a

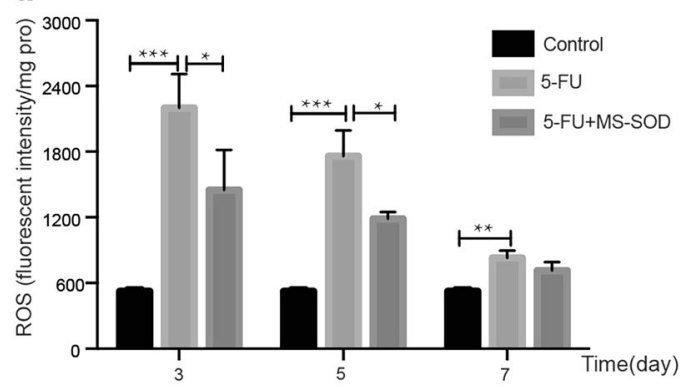

b

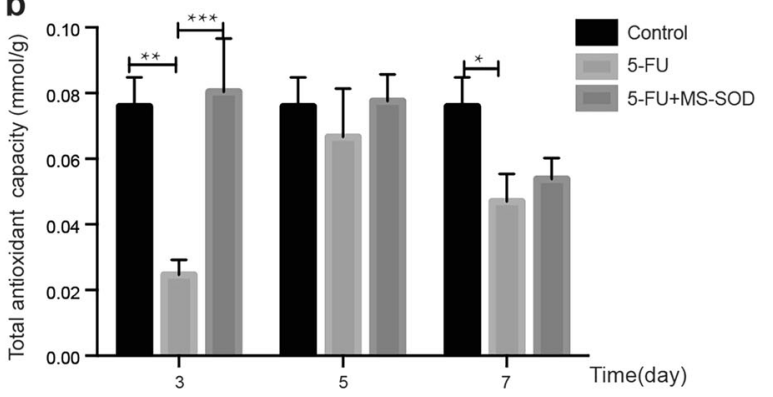

C
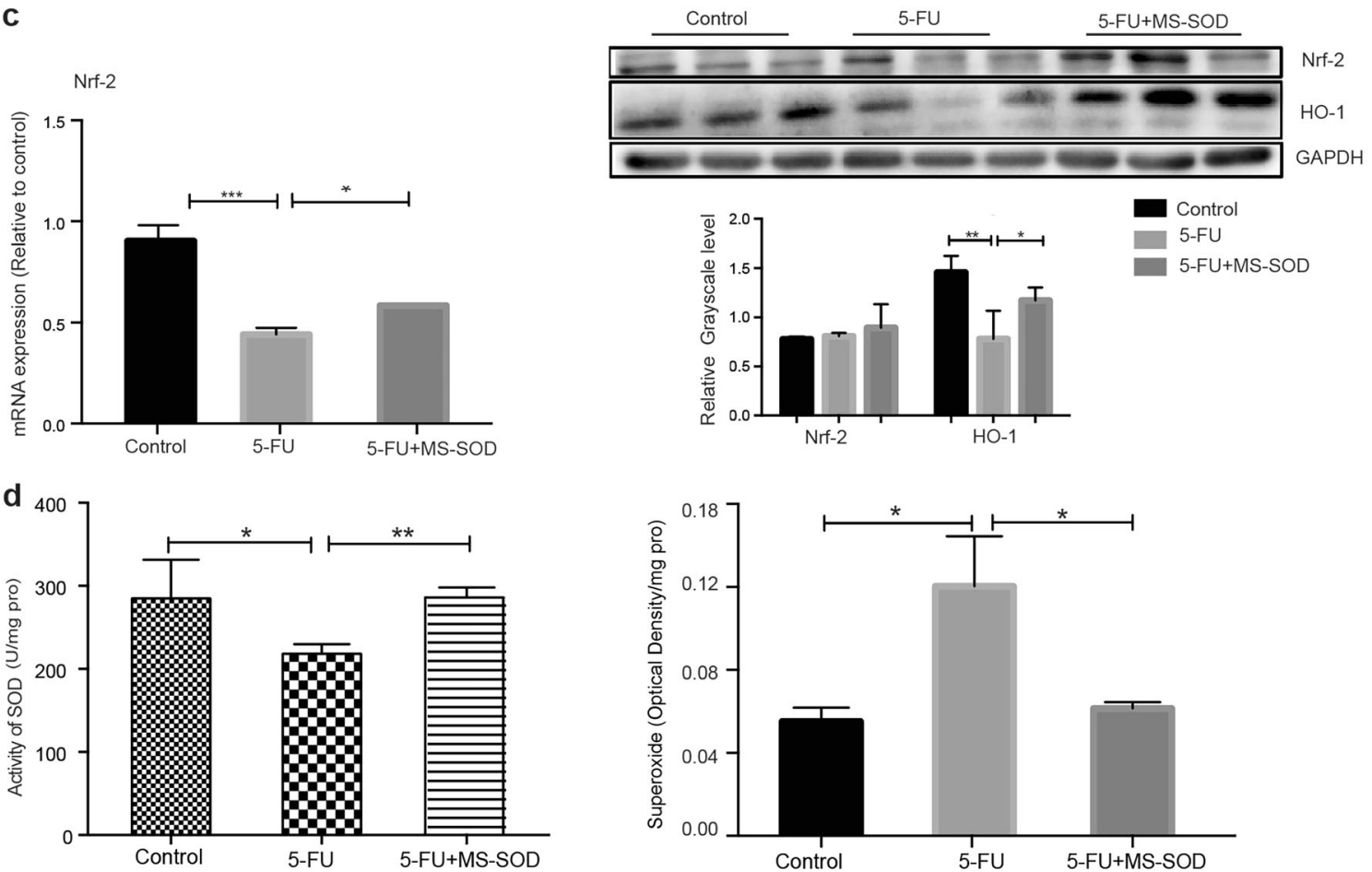

Fig. 4 Effects of MS-SOD on ROS levels and antioxidant capacity in the small intestine during 5-FU treatment. The ROS content and total antioxidant capacity of the small intestine at different times were detected using commercial kits (a, b). qPCR analysis was performed to measure the transcription level of Nrf2. Western blotting was performed to measure the level of HO-1 and Nrf2 translation (c). Activity of SOD and the content of superoxide were detected via kits (d). MS-SOD reduced the ROS content and increased the total antioxidant capacity in the small intestine during 5-FU injection. MS-SOD increased both Nrf2 and HO-1 expression in the small intestine after 5 days of 5-FU treatment. MS-SOD also increased the activity of SOD and decreased the superoxide content. The data are shown as the means \pm SEM $(n=6)$. ${ }^{*} P<0.05$, ${ }^{* *} P<0.01,{ }^{* *} P<0.001$. In $(\mathbf{c})$, the repeated blots in each group indicate three individuals

is mainly produced in pathological conditions, which further damages cells [27]. Therefore, inhibiting iNOS expression may also be a target for the treatment of inflammation $[27,28]$.

\section{DISCUSSION}

$5-\mathrm{FU}$ is one of the most common chemotherapeutic agents used for the treatment of malignant tumors [29]. However, 5-FU frequently causes intestinal mucositis, which is accompanied by severe diarrhea [2]. The serious side effects of 5-FU impair patient quality of life and might lead to the discontinuation of cancer chemotherapy. Therefore, effective countermeasures against 5FU-induced intestinal mucositis are necessary.

In the current study, we found that the novel SOD product MSSOD contributes to a reduction in 5-FU-induced intestinal mucositis. MS-SOD was constructed through genetic modification of the thermophilic HB27-encoded SOD gene and expressed in competent E. coli BL21 for batch production [15]. Compared with the reported products of thermophilic bacteria extraction, MS-SOD has a comprehensive advantage in its resistance to high temperature and pepsin and trypsin treatment, which precludes the generation of oral degradation fragments and maintains MSSOD activity after its oral administration [15]. Therefore, MS-SOD has been proposed as a therapeutic agent targeted to the intestine with demonstrated efficacy in the treatment of 5-FUinduced mucositis.

Several studies have demonstrated that the administration of 5-FU to experimental animals produces diarrhea and decreases body weight, accompanied by morphological damage to the intestine [16]. Our study indicated that the repeated administration of $5-\mathrm{FU}(50 \mathrm{mg} / \mathrm{kg})$ to mice causes severe intestinal mucositis. This mucositis is morphologically characterized by shortening of the villus height and the destruction of crypts in the small intestine. Once-daily administration of MS-SOD reduced the severity of 5-FU-induced morphological intestinal damage and clinical symptoms, including diarrhea and body weight loss. 


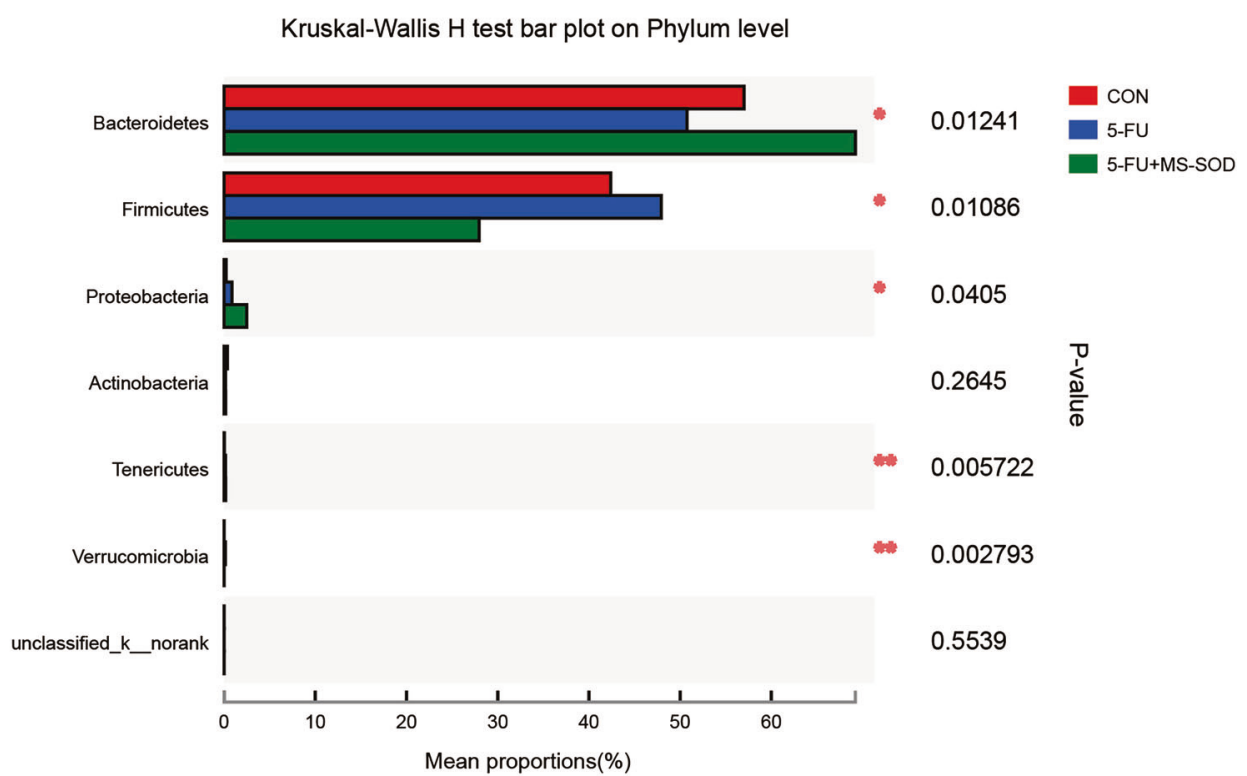

\section{b}

norank_f_Bacteroidales_S24-7_group

Kruskal-Wallis $\mathrm{H}$ test bar plot on Genus level

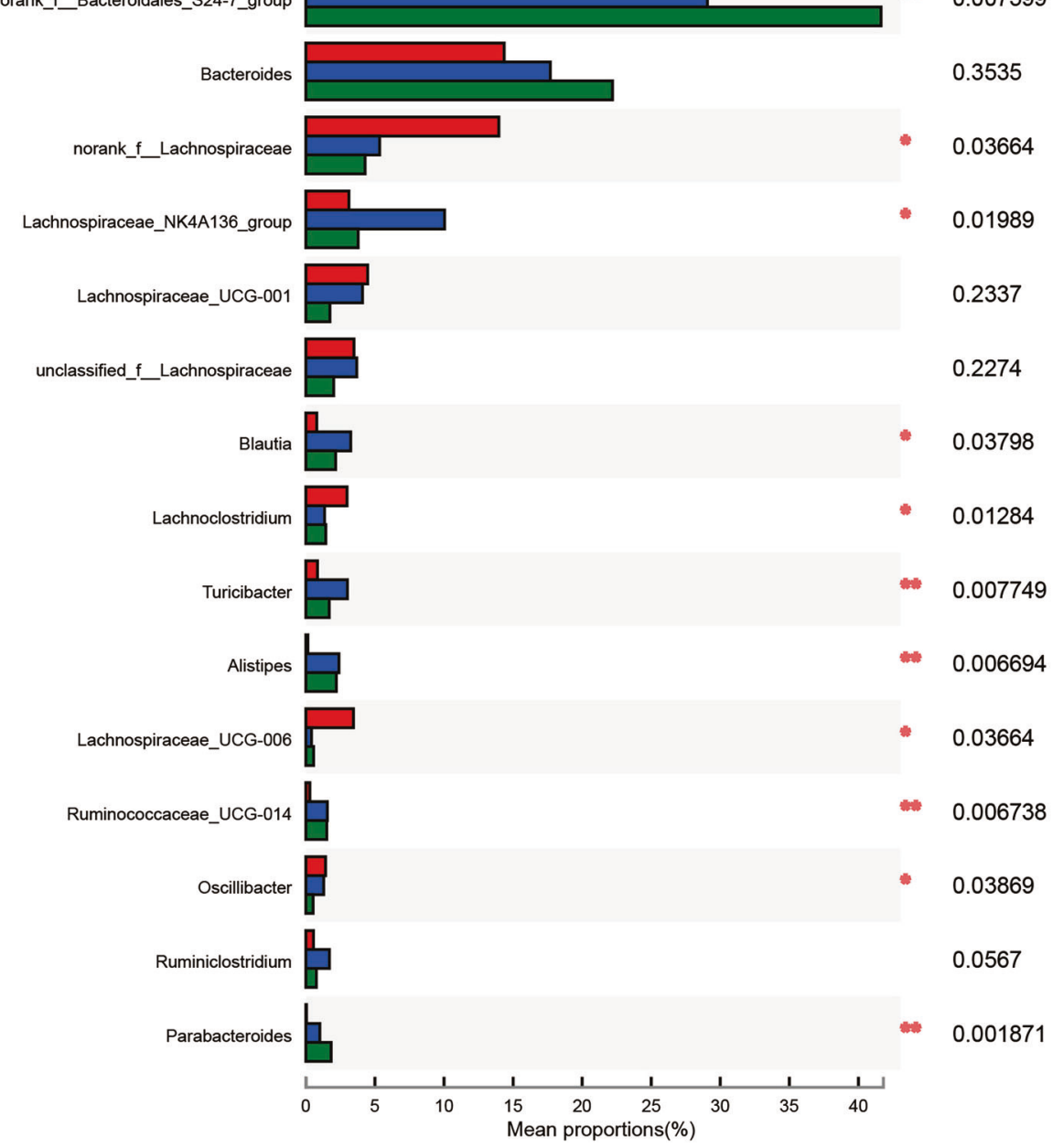

Fig. 5 Effects of MS-SOD on gut microbes after 5-FU treatment. Cecum feces were collected on day 6, and the relative abundance of microbes was analyzed via next-generation sequencing of bacterial 16S DNA. At the phylum level, 5-FU treatment decreased the abundance of Bacteroides but increased the abundance of Lachnospiraceae and Verrucomicrobia compared with the control mice. MS-SOD reversed this situation (a). At the genus level, more detailed changes were observed (b) 
Table 2. Effect of MS-SOD on 5-FU-induced changes in mouse bacterial flora

\begin{tabular}{lclllcc}
\hline & Bacteroidetes & Firmicutes & Proteobacteria & Actinobacteria & Tenericutes & Verrucomicrobia \\
\hline Control & $57 \pm 9.42$ & $42.39 \pm 9.51$ & $0.22 \pm 0.13$ & $0.35 \pm 0.21$ & $0.02 \pm 0.02$ & $0 \pm 0$ \\
5-FU & $50.76 \pm 10.24$ & $47.93 \pm 10.64$ & $0.86 \pm 0.75$ & $0.16 \pm 0.11$ & $0.16 \pm 0.067^{\# \#}$ & $0.12 \pm 0.17^{\#}$ \\
5-FU + MS-SOD & $69.21 \pm 6.50^{*}$ & $27.95 \pm 5.76^{*}$ & $2.48 \pm 4.77$ & $0.18 \pm 0.11$ & $0.17 \pm 0.15$ & $0 \pm 0^{*}$ \\
\hline
\end{tabular}

Animals were administered $50 \mathrm{mg} / \mathrm{kg} 5$-FU via intraperitoneal injection once daily for 5 days (days 1 to 5 ), with or without $800 \mathrm{U} / 10 \mathrm{~g}$ MS-SOD coadministered daily. Feces were collected on day 6 , and bacterial $16 \mathrm{~S}$ rDNA was quantified via PCR. The data are shown as the mean (\%) \pm SD (\%) values from 6 animals per group. ${ }^{\#} P<0.05,{ }^{\# \#} P<0.01$ vs. control; ${ }^{*} P<0.05,{ }^{* *} P<0.01$ vs. animals treated with 5 -FU only

According to the community abundance, species with significant differences in richness in microbial communities between different groups were found. At the phylum level, the relative abundance of Bacteroides in MS-SOD-treated mice was higher than that in the model group $(P<0.05)$, and the relative abundance of Firmicutes was lower than that in the model group $(P<0.05)$. In addition, The Verrucomicrobia population was significantly increased in the model group $(P<0.05)$ and was significantly decreased after MS-SOD administration $(P<0.01)$

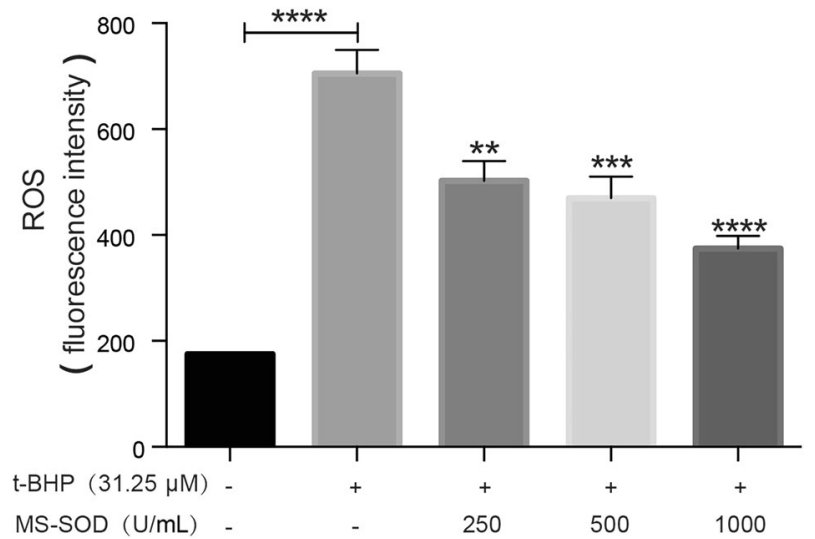

Fig. 6 In vitro activity of MS-SOD in tBHP treated Caco-2 cells. Cells were treated with $31.25 \mu \mathrm{M}$ tert-butyl hydroperoxide (tBHP) for $1 \mathrm{~h}$, and MS-SOD at the indicated concentrations were also added simultaneously. Cells were incubated for $30 \mathrm{~min}$ with $25 \mu \mathrm{M} 2^{\prime}, 7^{\prime}-$ dichlorofluorescein diacetate (DCFH-DA) and harvested for ROS content detection. tBHP induced an increase in ROS generation in Caco2 cells, but MS-SOD significantly decreased tBHP-induced ROS generation in a dose-dependent manner. ${ }^{* *} P<0.01,{ }^{* *} P<0.001$

5-FU-induced diarrhea is closely associated with intestinal mucositis severity, and deteriorating systemic conditions followed by diarrhea and dehydration which result in body weight loss [16]. Therefore, MS-SOD could be effective against 5-FU-induced intestinal mucositis and its related clinical symptoms. Because the intestinal epithelium renews every 3-5 days [30], mice injected with 5-FU once daily for 5 days showed severe mucositis gradually during the initial stage of the experiment but exhibited selfrenewal after the withdrawal of 5-FU. The administration of MSSOD alleviated the mucositis caused by 5 -FU primarily in the first 3-5 days; we chose animals that were treated for 5 days for detailed studies because MS-SOD directly scavenges superoxide radicals and because the initial injury factor and effects of superoxide radicals occur predominant early in disease progression. We did not emphasize the recovery stage in the mice because of the ability of the intestinal epithelium to renew. The mice appeared to experience faster recovery after the withdrawal of 5 -FU due to the effects of MS-SOD.

Although the pathogenesis of 5-FU-induced intestinal mucositis is not fully understood, several pathogenic elements in addition to its direct cytotoxicity are thought to be involved; these elements include ROS, abnormal inflammation and intestinal flora disorder [11]. ROS induction in particular is the initial event in intestinal mucositis induced by 5-FU [11]. Then, excess ROS levels activate a cascade of inflammatory pathways. ROS overproduction and inflammation destroy the antioxidant system, and ROS cannot be scavenged in time, which causes oxidative stress and cell damage.
The loss of mucosal integrity results in notably painful lesions that are prone to superficial bacterial colonization. These breaks in the mucosa serve as portals of entry for numerous microorganisms.

Our study determined the overproduction of ROS after the administration of 5 -FU. These findings suggest that ROS are important in the initiation of 5-FU-induced intestinal mucositis. ROS levels were significantly lower after the administration of MSSOD. MS-SOD attenuated oxidative stress in response to 5-FU and induced the antioxidant HO-1-Nrf2 pathway. Thus, MS-SOD significantly reduces 5 -FU-induced intestinal mucositis via inhibiting oxidative stress in intestinal crypts (Fig. 8).

Indeed, several studies have demonstrated that 5-FU-induced apoptosis can be potently prevented by inhibiting cytokine expression. In the current study, 5-FU administration caused the upregulation of cytokines related to inflammation and diarrhea, IL-6, IL-17a, IL-22, CXCL1 and CXCL2, in the small intestine. Interestingly, MS-SOD significantly attenuated the upregulation of these cytokines. We also detected the concentration of cytokines in both the serum and intestine, but most of these factors were difficult to detect in the intestine. Therefore, we chose to detect mRNA expression, and most of the cytokines in the intestine did not exhibit differences in expression between the 5-FU-treated mice and MSSOD-treated mice. Indeed, orally administered MS-SOD did not circulate in the blood and thus might have no effect on hematologic indexes (Supplementary Fig. S2).

Basic and clinical studies have demonstrated that cancer chemotherapy induces dysbiosis [18]. We also examined the influence of 5-FU and MS-SOD on the intestinal flora in mice. 5-FU decreased the relative abundance of Bacteroides and increased the relative abundance of Lachnospiraceae compared with their abundance in the control group. 5-FU treatment also increased the abundance of Verrucomicrobia. Akkermansia muciniphila, which belongs to the phylum Verrucomicrobia, is well known to degrade mucin [31] and promote intestinal inflammation. Interestingly, MS-SOD treatment reversed the changes in the 5-FUtreated mice reported above. Therefore, MS-SOD likely reduces 5 FU-induced mucositis by influencing the intestinal flora.

Furthermore, in vitro studies indicated that MS-SOD inhibited iNOS generation induced by LPS in a macrophage cell line and tBHP in Caco2 cells. iNOS is mainly produced during pathological states and may be induced by inflammatory substances, thus leading to a high level of NO production, further damaging cells and worsening inflammation. Therefore, NOS, the target of MSSOD, is inhibited by MS-SOD to treat inflammatory reactions. Mucosal damage is accompanied by barrier damage, including mechanical barrier, chemical barrier, biological barriers and immune barrier damage, among which mechanical barrier damage is the most important. The intestinal barrier structure depends on tight junctions (TJs) and adhesion proteins [32]. TJs are closely related to the development of intestinal diseases [32], and surface bacteria colonization is facile when there are cracks in 

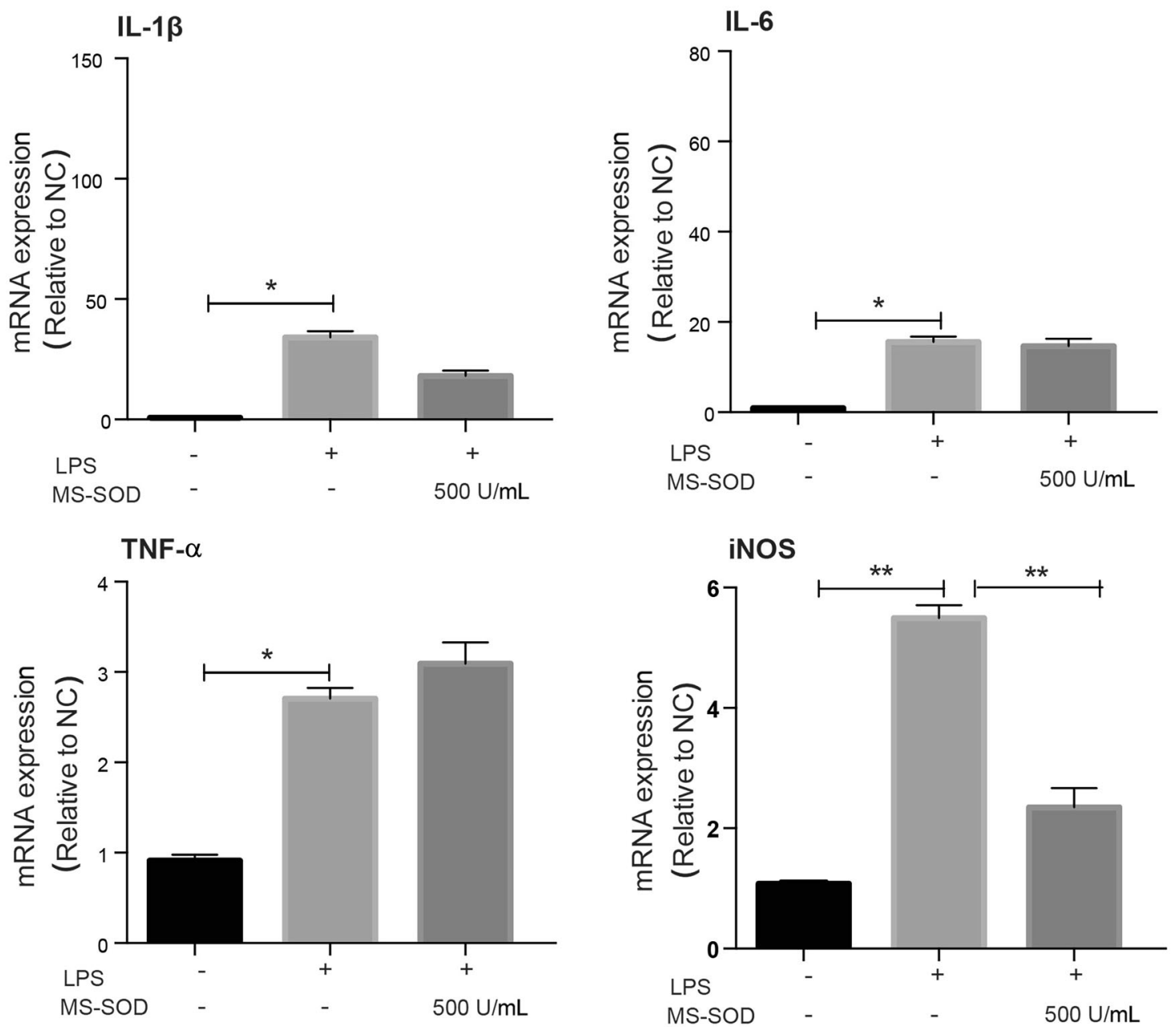

Fig. 7 In vitro activity of MS-SOD in LPS-treated RAW264.7 cells. RAW264.7 cells were pre-incubated with 500 U/mL MS-SOD for $6 \mathrm{~h}$, then stimulated with $10 \mathrm{~g} / \mathrm{mL}$ LPS and samples were collected $6 \mathrm{~h}$ after LPS stimulation, IL-1 $\beta$, IL- 6 , TNF-a and iNOS mRNA expression levels were measured with qPCR. LPS induced an increase in ROS generation in RAW264.7 cells, but MS-SOD significantly decreased LPS-induced ROS generation. ${ }^{*} P<0.05,{ }^{* *} P<0.01$

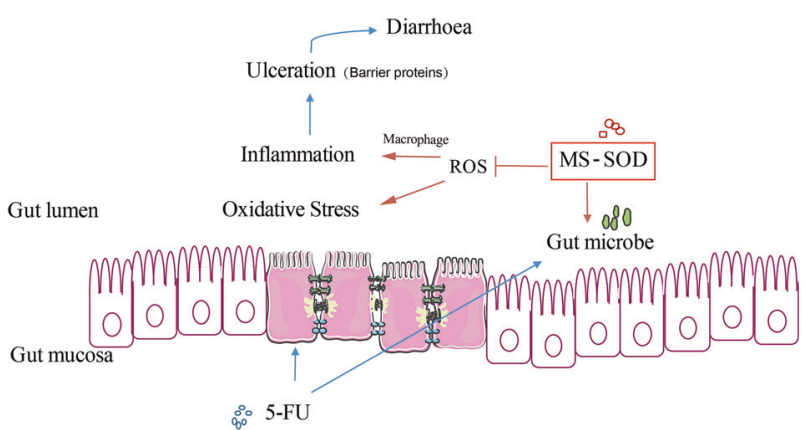

Fig. 8 Possible mechanism by which MS-SOD reduces intestinal injury in mice with 5 -FU-induced mucositis. The novel SOD product MS-SOD demonstrated efficacy in treatment of 5-FU-induced intestinal mucositis in this study. MS-SOD exerted effects through the following three main aspects. First, MS-SOD reduced ROS levels at the early stage of mucositis, thus contributing to inhibition of oxidative stress in intestinal crypts. Second, MS-SOD inhibited the expression of select cytokines associated with inflammation in the intestine. Third, MS-SOD changed the relative abundance of intestinal flora in the model mice. These three factors are relevant in the pathogenesis of mucositis, and MS-SOD improved these factors, suggesting several possible mechanisms by which MS-SOD can reduce mucositis the mucosa. The connected structural proteins include transmembrane proteins, cytoplasmic proteins and cytoskeletal proteins. These transmembrane proteins primarily include occludin [33, 34], claudins, and junction adhesion molecule (JAM). Claudin-2 is unregulated in the small and large intestine, where it contributes to diarrhea via a leak flux mechanism [34]. The expression of most TJ proteins was low after 5-FU administration. However, MS-SOD did not obviously protect these proteins. Therefore, we hypothesized that 5-FU destroyed the composition of these proteins through damage to the intestinal mucosa epithelium, while MSSOD failed to protect these proteins.

A model proposed by Sonis has been widely cited to explain the basic pathophysiology of mucosal injury [10]. When inflammation occurs, the antioxidant system is destroyed, and ROS cannot be cleared in time, leading to oxidative stress and damage to intracellular targets. Proinflammatory cytokines are released after their activation by chemotherapy and ROS, leading to tissue damage and apoptosis. The release of a large number of proinflammatory cytokines enhances the entire inflammatory process and prolongs tissue damage. These effects primarily occur in the submucosa and basal epithelium. At this stage, although the tissue biology has changed, no obvious mucosal injury is observed clinically. Intestinal mucosal ulcers and atrophic changes are the apex events in tissue damage along with stem cell death. The integrity of the intestinal epithelium is destroyed, 
and its function is impaired. Patients usually have diarrhea at this stage. In the present study, we propose that the main protective effect of MS-SOD against intestinal mucosal injury is its effective scavenging of ROS at the initial stage of mucosal injury and that MS-SOD thus plays an indirect role in inflammation and barrier protein protection, thereby reducing the degree of intestinal mucosal injury.

In conclusion, we found that MS-SOD reduced 5-FU-induced mucositis in mice by improving oxidative stress, inflammation and dysbiosis. Therefore, MS-SOD might have clinical benefits for the prevention of intestinal mucositis during cancer chemotherapy. However, it is important to investigate the effects of MS-SOD on mucositis induced by other chemotherapy drugs to reach a more convincing conclusion that MS-SOD is an effective treatment for intestinal mucositis in chemotherapy.

\section{ACKNOWLEDGEMENTS}

This study was supported by the National New Drug Creation Program of China (2015ZX09501007-001 to Jian-hua Sun and 2018ZX09201017-004 to Li-kun Gong) and the "Strategic Priority Research Program" of the Chinese Academy of Sciences (XDA12050305 to Li-kun Gong).

\section{AUTHOR CONTRIBUTIONS}

$X X Y, Y T Z, F G M$ and LKG designed the research and wrote the manuscript. XXY, HLL, SYW, XLY, and JHS conducted the experiments. HLL contributed to the pathological analysis, and YTZ performed data analysis.

\section{ADDITIONAL INFORMATION}

The online version of this article (https://doi.org/10.1038/s41401-019-0295-8) contains supplementary material, which is available to authorized users.

Competing interests: The authors declare no competing interests.

\section{REFERENCES}

1. Rosenthal DI, Mendoza TR, Fuller CD, Hutcheson KA, Wang XS, Hanna EY, et al. Patterns of symptom burden during radiotherapy or concurrent chemoradiotherapy for head and neck cancer: a prospective analysis using the university of Texas MD anderson cancer center symptom inventory-head and neck module. Cancer. 2014;120:1975-84.

2. Harris DJ. Cancer treatment-induced mucositis pain: strategies for assessment and management. Ther Clin Risk Manag. 2006;2:251-8.

3. Swami U, Goel S, Mani S. Therapeutic targeting of CPT-11 induced diarrhea: a case for prophylaxis. Curr Drug Targets. 2013;14:777-97.

4. lacovelli R, Pietrantonio F, Palazzo A, Maggi C, Ricchini F, de Braud F, et al. Incidence and relative risk of grade 3 and 4 diarrhoea in patients treated with capecitabine or 5-fluorouracil: a meta-analysis of published trials. $\mathrm{Br} \mathrm{J}$ Clin Pharmacol. 2014;78:1228-37.

5. Simone NL, Menard C, Soule BP, Albert PS, Guion P, Smith S, et al. Intrarectal amifostine during external beam radiation therapy for prostate cancer produces significant improvements in quality of life measured by epic score. Int J Radiat Oncol Biol Phys. 2008;70:90-5.

6. Zhou YF, Sridhar R, Shan L, Sha W, Gu XB, Sukumar S. Loperamide, an FDAapproved antidiarrhea drug, effectively reverses the resistance of multidrug resistant MCF-7/MDR1 human breast cancer cells to doxorubicin-induced cytotoxicity. Cancer Invest. 2012;30:119-25.

7. Deng C, Deng B, Jia LQ, Tan HY. Efficacy of long-acting release octreotide for preventing chemotherapy-induced diarrhoea: protocol for a systematic review. BMJ Open. 2017;7:e014916.

8. Huang JH, Cao YF, Liao C, Wu LC, Gao F. Effect of histamine-2-receptor antagonists versus sucralfate on stress ulcer prophylaxis in mechanically ventilated patients: a meta-analysis of 10 randomized controlled trials. Crit Care. 2010;14:R194.

9. Aggarwal BB, Prasad S, Reuter S, Kannappan R, Yadav VR, Park B, et al. Identification of novel anti-inflammatory agents from ayurvedic medicine for prevention of chronic diseases: "Reverse Pharmacology" and "Bedside to Bench" approach. Curr Drug Targets. 2011;12:1595-653.

10. Sonis ST. The pathobiology of mucositis. Nat Rev Cancer. 2004;4:277-84.
11. Bhattacharyya A, Chattopadhyay R, Mitra S, Crowe SE. Oxidative stress: an essential factor in the pathogenesis of gastrointestinal mucosal diseases. Physiol Rev. 2014;94:329-54.

12. Zhang XC, Epperly MW, Kay MA, Chen ZY, Dixon T, Franicola $D$, et al. Radioprotection in vitro and in vivo by minicircle plasmid carrying the human manganese superoxide dismutase transgene. Hum Gene Ther. 2008;19:820-6.

13. Ahlengard $S$, Tufesson $G$, Pettersson $H$, Andersson $T$. Treatment of traumatic arthritis in the horse with Intra-Articular Orgotein (Palosein"). Equine Vet J. 1978;10:122-4.

14. Goldberg LD, Crysler C. A single center, pilot, double-blinded, randomized, comparative, prospective clinical study to evaluate improvements in the structure and function of facial skin with tazarotene $0.1 \%$ cream alone and in combination with GliSODin ${ }^{\circledast}$ skin nutrients advanced anti-aging formula. Clin Cosmet Invest Dermatol. 2014;7:139-44.

15. Meng FG, inventors. Novel recombinant high-stability superoxide dismutase and application thereof. China patent. 2016;ZL201610099824.

16. Hamouda N, Sano T, Oikawa Y, Ozaki T, Shimakawa M, Matsumoto K, et al. Apoptosis, dysbiosis and expression of inflammatory cytokines are sequential events in the development of 5-fluorouracil-induced intestinal mucositis in mice. Basic Clin Pharmacol Toxicol. 2017;121:159-68.

17. Yasuda $M$, Kato $S$, Yamanaka $N$, limori $M$, Matsumoto $K$, Utsumi $D$, et al. 5-HT3 receptor antagonists ameliorate 5 -fluorouracil-induced intestinal mucositis by suppression of apoptosis in murine intestinal crypt cells. $\mathrm{Br} J$ Pharmacol. 2013;168:1388-400.

18. Li HL, Lu L, Wang XS, Qin LY, Wang P, Qiu SP, et al. Alteration of gut microbiota and inflammatory cytokine/chemokine profiles in 5-fluorouracil induced intestinal mucositis. Front Cell Infect Microbiol. 2017;7:455.

19. Liu ZZ, Xi J, Schroder S, Wang WG, Xie TP, Wang ZG, et al. Chimonanthus nitens var. salicifolius aqueous extract protects against 5 -fluorouracil induced gastrointestinal mucositis in a mouse model. Evid Based Complement Altemat Med. 2013;2013:789263.

20. Wright TH, Yazbeck R, Lymn KA, Whitford EJ, Cheah KY, Butler RN, et al. The herbal extract, lberogast $(R)$, improves jejunal integrity in rats with 5 -fluorouracil (5-FU)-induced mucositis. Cancer Biol Ther. 2009;8:923-9.

21. Al-Sadi R, Khatib K, Guo SH, Ye DM, Youssef M, Ma T. Occludin regulates macromolecule flux across the intestinal epithelial tight junction barrier. Am J Physiol Gastrointest Liver Physiol. 2011;300:G1054-64.

22. Lin $Y$, Lin $Y$, Lin L, Zheng CQ. IL-17/IFN-gamma interactions regulate intestinal inflammation in TNBS-induced acute colitis. J Interferon Cytokine Res. 2012;32:548-56.

23. Hosseini MAS, Mansourabadi AH, Shams A, Hassanzadeh M. The role of interleukin (IL-22) in immune response to human diseases. Int J Epidemiol Res. 2015;2:152-61.

24. Sakai H, Kai $Y$, Oguchi $A$, Kimura $M$, Tabata $S$, Yaegashi $M$, et al. Curcumin inhibits 5-fluorouracil-induced up-regulation of CXCL1 and CXCL2 of the colon associated with attenuation of diarrhoea development. Basic Clin Pharmacol Toxicol. 2016;119:540-7.

25. Xu L, He SS, Yin P, Li DY, Mei C, Yu XH, et al. Punicalagin induces Nrf2 translocation and $\mathrm{HO}-1$ expression via $\mathrm{PI} 3 \mathrm{~K} / \mathrm{Akt}$, protecting rat intestinal epithelial cells from oxidative stress. Int J Hyperther. 2016;32:465-73.

26. Asakura H, Suzuki K, Kitahora T, Morizane T. Is there a link between food and intestinal microbes and the occurrence of Crohn's disease and ulcerative colitis? J Gastroenterol Hepatol. 2008;23:1794-801.

27. Forstermann U, Sessa WC. Nitric oxide synthases: regulation and function. Eur Heart J. 2012;33:829-37.

28. Hernandez-Saavedra D, Zhou HF, McCord JM. Anti-inflammatory properties of a chimeric recombinant superoxide dismutase: SOD2/3. Biomed Pharmacother. 2005;59:204-8.

29. Longley DB, Harkin DP, Johnston PG. 5-Fluorouracil: mechanisms of action and clinical strategies. Nat Rev Cancer. 2003;3:330-8.

30. van der Flier LG, Clevers H. Stem cells, self-renewal, and differentiation in the intestinal epithelium. Annu Rev Physiol. 2009;71:241-60.

31. Ganesh BP, Klopfleisch R, Loh G, Blaut M. Commensal Akkermansia muciniphila exacerbates gut inflammation in Salmonella typhimurium-infected gnotobiotic mice. PLoS One. 2013;8:e74963.

32. Hu CA, Hou Y, Yi D, Qiu Y, Wu G, Kong $X$, et al. Autophagy and tight junction proteins in the intestine and intestinal diseases. Anim Nutr. 2015;1:43-7.

33. Weber CR, Shen L, Wu LC, Wang YM, Turner JR. Occludin is required for tumor necrosis factor (TNF)-mediated regulation of tight junction (TJ) barrier function. Gastroenterology. 2011;140:S64.

34. Luettig J, Rosenthal R, Barmeyer C, Schulzke JD. Claudin-2 as a mediator of leaky gut barrier during intestinal inflammation. Tissue Barriers. 2015;3:e977176. 